\title{
Productos comunicacionales para la prevención de la COVID-19 promovidos por los gobiernos de América Latina y el Caribe
}

\author{
Daniela Moyano ${ }^{1}$ y Lina Lay Mendivil ${ }^{2}$
}

Forma de citar

Moyano D, Lay Mendivil L. Productos comunicacionales para la prevención de la COVID-19 promovidos por los gobiernos de América Latina y el Caribe. Rev Panam Salud Publica. 2021;45:e111. https://doi.org/10.26633/RPSP.2021.111

RESUMEN

Objetivo. Caracterizar el contenido de los productos comunicacionales dirigidos a la población general para la prevención de la COVID-19 promovidos por los gobiernos de América Latina y el Caribe (ALC).

Métodos. Estudio descriptivo, trasversal y exploratorio de los productos comunicacionales publicados hasta el 28 de febrero del 2021 en los sitios web oficiales de los ministerios de salud de los 47 países y territorios de ALC para la prevención de la COVID-19. Se emplearon métodos cuantitativos y cualitativos a partir de dimensiones recomendadas por la Organización Mundial de la Salud (OMS) y un enfoque de determinantes sociales de la salud (DSS). Se utilizó la técnica de análisis de contenido y triangulación por observador.

Resultados. Se analizaron 3253 productos informativos; en los 47 países y territorios de ALC, al menos un producto incluyó alguna recomendación de la OMS y en 46 se abordó alguna de las categorías y subcategorías relacionadas con los DSS intermedios. De los DSS estructurales, se aplicó el enfoque de género y alguna lengua indígena en al menos un producto de solo 4 y 7 países, respectivamente. En 35 países se encontró al menos un producto con información errónea y en 13 no se abordó la infodemia.

Conclusiones. Se encontró una cantidad considerable de productos comunicacionales para la prevención de la COVID-19, pero no reflejaban un adecuado enfoque de diversidad y salud integral, y mostraban vacíos y errores de comunicación. Los países deben revisar sus políticas de comunicación en salud en el contexto de la pandemia.

Palabras clave Comunicación en salud; infecciones por coronavirus; programas de gobierno; América Latina; región del Caribe.

Desde la notificación del primer caso de COVID-19 en América Latina y el Caribe (ALC), esta enfermedad se ha propagado a todos los países y territorios del área (1) en un contexto en el que la desigualdad en el acceso a los servicios de salud sigue siendo un problema estructural (2).

Para disminuir las desigualdades es fundamental que la información relacionada con la salud sea adecuada, suficiente, clara, oportuna y accesible (3). Especialmente durante las crisis sanitarias, la comunicación en temas de salud influye en la toma de decisiones de las personas y, si los materiales comunicacionales son adecuados, pueden propiciar los cambios de comportamiento necesarios (4).

La pandemia de COVID-19 ha generado una explosión global de información (5), para lo que se ha acuñado el término infodemia: exceso abrumador de información, con una considerable presencia de datos inexactos, falsos o engañosos (6). En este contexto es imprescindible profundizar en el contenido de las campañas de comunicación para ayudar a contener la

\footnotetext{
Universidad Nacional de La Matanza, Buenos Aires, Argentina. Universidad Nacional de Córdoba, Córdoba, Argentina. $₫$ Daniela Moyano, moyanodaniela12@gmail.com
} 
propagación de informaciones sin fundamento científico y contribuir al manejo de la pandemia desde una correcta comunicación de riesgo $(7,8)$, algo estrechamente relacionado con la alfabetización en salud (9).

Investigaciones previas sobre la comunicación a la población durante la pandemia indican que varios medios y portales informativos compartieron información falsa (10) o inexacta (11), cuyo impacto negativo resulta mayor en grupos vulnerables, que luego sufren graves consecuencias a causa de esa mala información (12). Por otro lado, existe la preocupación de que los mensajes emitidos durante la pandemia no estén llegando a una parte importante de la población en riesgo (13). Los materiales comunicacionales y los mensajes deben estar diseñados de modo que nadie quede excluido (14).

Si bien existen antecedentes científicos sobre comunicación de riesgo durante los brotes de ébola, zika y fiebre amarilla (15), faltan investigaciones concretas sobre el contenido de las campañas de comunicación generadas desde los gobiernos de ALC durante la actual pandemia de COVID-19.

En este trabajo se hace una caracterización del contenido de los productos comunicacionales dirigidos a la población general para la prevención de la COVID-19 promovidos por los gobiernos de ALC.

\section{MATERIALES Y MÉTODOS}

Se realizó un estudio descriptivo, trasversal y exploratorio de los productos comunicacionales publicados en sitios web oficiales de los gobiernos de ALC (16), con un enfoque centrado en la prevención, según las recomendaciones específicas para el público general (17), la comunicación de riesgo (8) y los determinantes sociales de la salud (DSS) (18).

Se emplearon métodos cuantitativos y cualitativos; las unidades de análisis se seleccionaron de manera intencional, según criterios de inclusión y exclusión preestablecidos.

Criterios de inclusión: todos los productos comunicacionales y el contenido de sus mensajes relacionados con la prevención de la COVID-19 y el contexto de la pandemia destinados a la población general. Se consideraron los materiales alojados en los sitios web de los ministerios de salud de los 47 países y territorios (incluidos los departamentos de ultramar) de ALC (19), publicados desde el comienzo de la pandemia en cada país y que se encontraban disponibles y accesibles hasta el 28 de febrero del 2021. Se seleccionaron todos los productos presentes y accesibles en la web, sin distinción de formato y origen, en español, inglés, portugués, neerlandés y francés, según el idioma oficial de cada país.

En el caso de las redes sociales (Facebook y YouTube) y otros sitios oficiales de gobierno, solo se incluyeron los productos que tuvieran acceso directo desde las páginas de los ministerios de salud.

Cuando se constató un número muy reducido o nulo de productos comunicacionales en el sitio de Internet de la entidad gubernamental encargada de la salud, se incluyeron los productos contenidos en páginas gubernamentales específicas sobre la COVID-19 y otras páginas de gobierno que tuvieran una sección para la COVID-19; en caso de no contar con productos comunicacionales en esas fuentes, se recurrió a la página oficial en Facebook de la autoridad sanitaria del país o territorio en cuestión.
Criterios de exclusión: productos comunicacionales y mensajes sobre prevención en el marco de la pandemia que estuvieran dirigidos exclusivamente a grupos específicos (niños, niñas, adolescentes, personas mayores, embarazadas, lactantes, personas de riesgo y con enfermedades preexistentes — crónicas o no-, pacientes en aislamiento, personas con síntomas y diagnóstico de COVID-19, deportistas o propietarios de animales domésticos, entre otros). También se excluyeron los productos destinados a centros escolares, y personal e instituciones de salud; documentos meramente normativos, epidemiológicos y sobre cobertura de vacunación; notas y comunicados de prensa, noticias, avisos y propaganda oficial; lineamientos, guías y orientaciones para la atención de pacientes y las pruebas de COVID-19; protocolos de bioseguridad específicos y recomendaciones destinados a instituciones educativas, comercios, restaurantes, gimnasios, eventos especiales y turísticos, transporte, bancos y empleos; blogs o mensajes de usuarios. Por último, se excluyeron los productos en lenguas indígenas, dialectos locales y otros idiomas no oficiales de ALC que no contaran con traducción a los idiomas oficiales incluidos en este estudio.

\section{Recolección de datos}

La recogida de datos se llevó a cabo mediante una búsqueda sistemática realizada durante el mes de febrero del 2021. Para mejorar la confiabilidad y la reproducibilidad del estudio se utilizaron las siguientes palabras clave en el motor de búsqueda de Google: COVID-19, nombre del ministerio en el idioma oficial del país y nombre del país. También se realizó una búsqueda manual.

Se empleó un instrumento de recolección de información en línea basado en dimensiones y categorías preestablecidas, con un campo abierto para la información emergente. Se descargaron y guardaron todos los productos comunicacionales de los sitios web explorados.

Para mejorar la validez interna y mitigar posibles sesgos, se revisó al menos dos veces toda la información recogida, se eliminaron productos duplicados y se llevó a cabo una triangulación por investigador (20). De esta manera, también se revisaron discrepancias y se corrigieron posibles errores en la entrada de datos.

\section{Variables, dimensiones y categorías}

Para el análisis cuantitativo se utilizaron las siguientes variables y categorías:

- Países de ALC según la subregión (19), con 3 categorías: América del Sur, América Central y México, y el Caribe.

- Tipo de producto (21), con 10 categorías: video (video de más de 30 segundos), spot (video de hasta 30 segundos), infografía, texto suelto, imagen con o sin texto, boletín, guía, audio, y material interactivo (enlaces a WhatsApp, teléfonos, chats en línea, buzón para sugerencias en línea, aplicaciones telefónicas o apps, y enlaces a teleconsultas).

- Tipo de soporte digital, con 4 categorías: sitio web del ministerio de salud (incluidos los enlaces a otras páginas de gobierno), del gobierno y específico sobre COVID-19 no perteneciente al área de salud, y página oficial de la autoridad sanitaria en Facebook. 
- Consejos para la prevención de la COVID-19 según las orientaciones de la Organización Mundial de la Salud (OMS) para el público general (17). Se definió como "presente" cuando en al menos un producto comunicacional se hacía mención, o se incorporaba — de manera total o parcial- alguna de las siguientes 15 categorías: lavado de las manos con agua y jabón; lavado de las manos con gel hidroalcohólico; uso de mascarilla casera; uso de mascarilla quirúrgica o médica; distanciamiento social de al menos un 1 metro; distanciamiento social de más de 1 metro; evitar espacios cerrados o congestionados; reuniones al aire libre; ventilación de espacios cerrados; forma sana de toser o estornudar: no tocarse la cara, nariz u ojos; limpieza y desinfección de superficies y objetos; conocer los síntomas; cómo hacer el autoaislamiento; y cómo y dónde buscar atención médica.

Las dimensiones empleadas en el análisis cualitativo fueron:

- DSS intermedios propuestos por la OMS (18). Se definió como "presente" cuando en al menos un producto comunicacional se hacía mención o se incorporaba — de manera total, parcial o trasversal- alguna de las siguientes 4 categorías y sus respectivas subcategorías: factores psicosociales (salud mental) -incluidos elementos de comunicación de riesgo a partir del análisis de la infodemia $(8,22)$-, circunstancias materiales (entorno), sistema de salud (vacunación contra la COVID-19), y factores biológicos y de comportamiento (hábitos saludables).

- DSS estructurales propuestos por la OMS (18), abordados desde un enfoque de diversidad $(23,24)$. Se definió como "presente" cuando en al menos un producto comunicacional se hacía mención o se incorporaba - de manera total, parcial o trasversal - alguna de las siguientes 4 categorías y sus respectivas subcategorías: inclusión de indígenas, afrodescendientes y migrantes; adaptación a diferentes lenguas, idiomas o dialectos; inclusión de personas con discapacidad; y enfoque de género.

\section{Análisis de los datos}

Para analizar las variables cuantitativas se utilizaron frecuencias absolutas y relativas. Para los datos cualitativos se aplicó el análisis de contenido en dos etapas (25): análisis global para la familiarización con la información, y análisis en profundidad según las categorías y subcategorías preestablecidas y emergentes; se identificaron elementos visuales, audiovisuales, frases, palabras del título y del cuerpo del mensaje, y se generaron descripciones de acuerdo con la naturaleza del producto comunicacional.

Todos los elementos identificados se clasificaron y codificaron mediante la técnica manual de codificación de la información con la ayuda del programa informático ATLAS.ti (versión 6.2).

Se seleccionaron ejemplos de productos y sus respectivos enlaces para ilustrar el contenido de las categorías (cuadros A1, A2 y A3 del material suplementario).

Este estudio se basó en productos comunicacionales publicados en sitios en Internet y redes sociales de entidades gubernamentales con acceso libre y abierto, y no se trabajó con seres humanos, por lo que tanto el Comité Nacional de Bioética de la Investigación de Panamá como el Comité Institucional de
Ética de las Investigaciones en Salud del Hospital Nacional de Clínicas de la Universidad Nacional de Córdoba de Argentina, dictaminaron su exención de evaluación.

\section{RESULTADOS}

Después de aplicar los criterios de inclusión y exclusión, se seleccionaron para el análisis 3253 productos comunicacionales, de ellos 1825 (56,1\%) se basaban en infografías, 532 (16,4\%) en videos de más de 30 segundos, $228(7,0 \%)$ en imágenes con texto, y el resto eran spots $(146 ; 4,5 \%)$, textos sueltos $(126 ; 3,9 \%)$, imágenes sin texto $(16 ; 0,5 \%)$, boletines $(42 ; 1,3 \%)$, guías $(148$; $4,5 \%)$, y materiales de audio $(160 ; 4,9 \%)$ e interactivos $(30 ; 0,9 \%)$, que se encontraron presentes en menos del $5 \%$ de los productos seleccionados.

Del total, 1044 (32,1\%) se encontraban en los sitios web de los ministerios o en sus enlaces a otros sitios gubernamentales, $1078(33,1 \%)$ en la red social Facebook oficial de los ministerios de salud, $598(18,4 \%)$ en otros sitios de gobierno y $533(16,4 \%)$ en sitios específicos de COVID-19 no pertenecientes al área de salud.

La mayor cantidad de productos informacionales se encontraron en sitios web de países de América Central y México (1 322; 40,7\%), seguidos por los del Caribe (1 172; 36,0\%) y América del Sur $(759 ; 23,3 \%)$.

\section{La prevención de la COVID-19 en las orientaciones al público general}

En todos los países y territorios de ALC se encontró al menos un producto comunicacional que incluyó algunas de las recomendaciones establecidas por la OMS (figura 1). La recomendación del lavado de manos con agua y jabón estuvo presente en al menos un producto en todos los países y territorios analizados.

La proporción de países que dieron la recomendación de mantener una distancia de más de 1 metro de otras personas en al menos un producto fue de $100 \%$ en América Central y México, de 84,0\% en el Caribe; y de 57,1\% en América del Sur; el resto de los países recomendaron el distanciamiento de al menos 1 metro.

Fue menor la proporción de países que abordaron en al menos un producto aspectos relacionados con el entorno, como las reuniones al aire libre: ninguno de América Central y México, el $20 \%$ en el Caribe y el 50\% en América del Sur (figura 1) (cuadro A1, del material suplementario).

\section{Determinantes intermedios}

En 46 países o territorios se abordó alguna de las categorías y subcategorías relacionadas con los DSS intermedios en al menos un producto comunicacional; dentro de la categoría de salud mental, la prevención de suicidio estuvo presente en al menos un producto de 4 países y dentro de la categoría de hábitos saludables, la prevención de enfermedades no transmisibles se encontró en materiales de 10 países (cuadro 1) (cuadro A2 del material suplementario).

Cabe destacar que en $35(74,5 \%)$ de los países estudiados hubo al menos un producto con información errónea y en 13 $(27,7 \%)$ no se encontró al menos un producto que abordara total o parcialmente la prevención de la infodemia (cuadro 1). 
FIGURA 1. Proporción de países con presencia de al menos un producto comunicacional promovido por los gobiernos que se ajusta a las medidas preventivas de la COVID-19a, según la subregión de América Latina y el Caribe, 2021

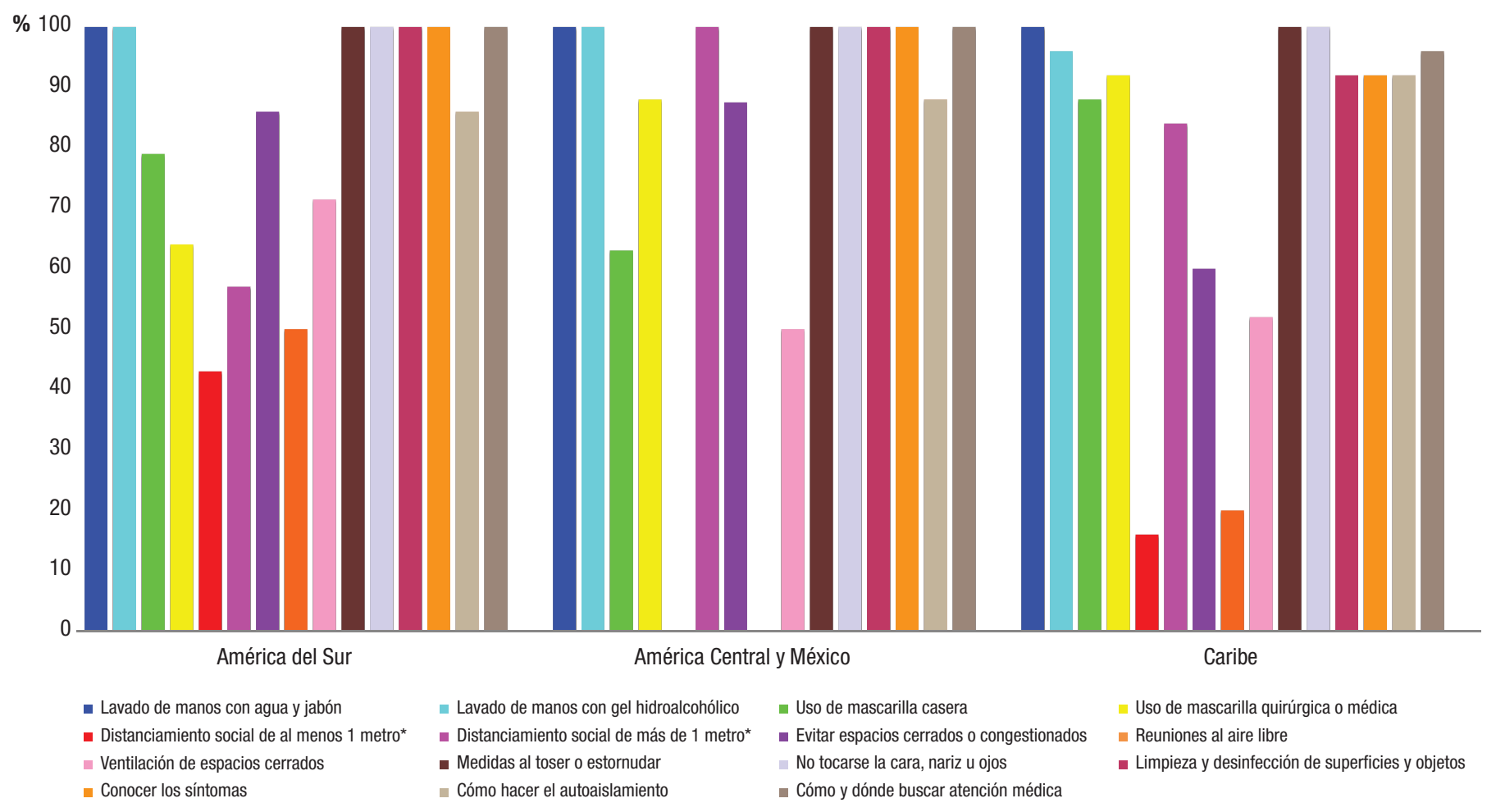

Fuente: elaboración propia.

Según las orientaciones de la Organización Mundial de la Salud para la prevención de la COVID-19 destinadas al público general (17).

Se definió como presencia cuando en al menos un producto comunicacional se hacía mención, o se incorporaba —de manera total, parcial o transversal—alguna de las medidas preventivas de la COVID-19.

En el caso de los países en los que se encontraron productos comunicacionales con recomendaciones de diferentes medidas de distanciamiento, ya fueran de al menos 1 metro (incluido cuando no se especificó la distancia) o de más de 1 metro, se consideró la medida que tuvo mayor presencia.

\section{Determinantes estructurales}

Como se muestra en el cuadro 2, a partir del análisis de los productos comunicacionales que aplicaban o incluían — de manera total, parcial o transversal- un enfoque de diversidad, se encontró que solo en 7 países se adaptó al menos un producto a lenguas indígenas, en 4 países se incluyó a personas indígenas en los mensajes y en 9 a personas con discapacidad; solo en 1 país se desarrollaron mensajes destinados a personas migrantes. Se observó que solo en 4 países se incluyó en al menos un producto alguna de las subcategorías definidas dentro del enfoque de género (cuadro A3 del material suplementario).

\section{DISCUSIÓN}

Si bien se encontraron productos comunicacionales que dentro de sus temáticas abordaron las recomendaciones de la OMS para la prevención de la COVID-19, se observó un limitado enfoque desde los DSS, en especial en lo relacionado con la diversidad y el abordaje integral de la salud. Es preocupante que se hayan encontrado productos con errores, así como la escasa presencia de productos y mensajes dirigidos a prevenir la infodemia.

Un estudio previo mostró que en la crisis sanitaria provocada por el virus del Ébola, el nivel de comunicación fue mínimo, con un uso reducido de materiales en formato de multimedia (26). En contraste, en el presente estudio se constató que los recursos utilizados se basaron en diferentes formatos digitales con predominio de infografías y videos de más de 30 segundos, algo similar a lo encontrado por Villegas-Tripiana y colaboradores en un estudio que analizó las campañas de comunicación sobre la COVID-19 en España (27).

En cuanto a las medidas preventivas de la COVID-19 abordadas, se encontró una fuerte presencia de productos y mensajes sobre el distanciamiento social, el lavado de las manos, el uso de mascarillas y la salud respiratoria, consistente con lo encontrado en el estudio español antes citado (27). Otra similitud con ese estudio es que, si bien se encontraron algunos productos con recomendaciones sobre salud mental, hábitos saludables y otras prácticas de prevención de enfermedades no transmisibles, estos tuvieron un alcance limitado. Como plantean Mheidly y Fares (28), el cierre de clínicas y la demora de los turnos pueden empeorar las condiciones crónicas de salud, por lo que en este contexto, las redes sociales serían una herramienta estratégica para promover la salud y el bienestar.

En la mayoría de los países se brindó algún tipo de información relacionada con la vacunación contra la COVID-19, más de lo encontrado por Villegas-Tripiana y colaboradores, que abordaron la vacunación general (27); esto puede deberse a que ese estudio se llevó a cabo en una etapa temprana de la pandemia, cuando todavía no se avizoraban las vacunas. Esta discrepancia muestra cómo en el entorno dinámico de la pandemia, las medidas implementadas por los gobiernos son cambiantes $\mathrm{y}$, por consiguiente, se deben modificar los mensajes comunicacionales difundidos. 

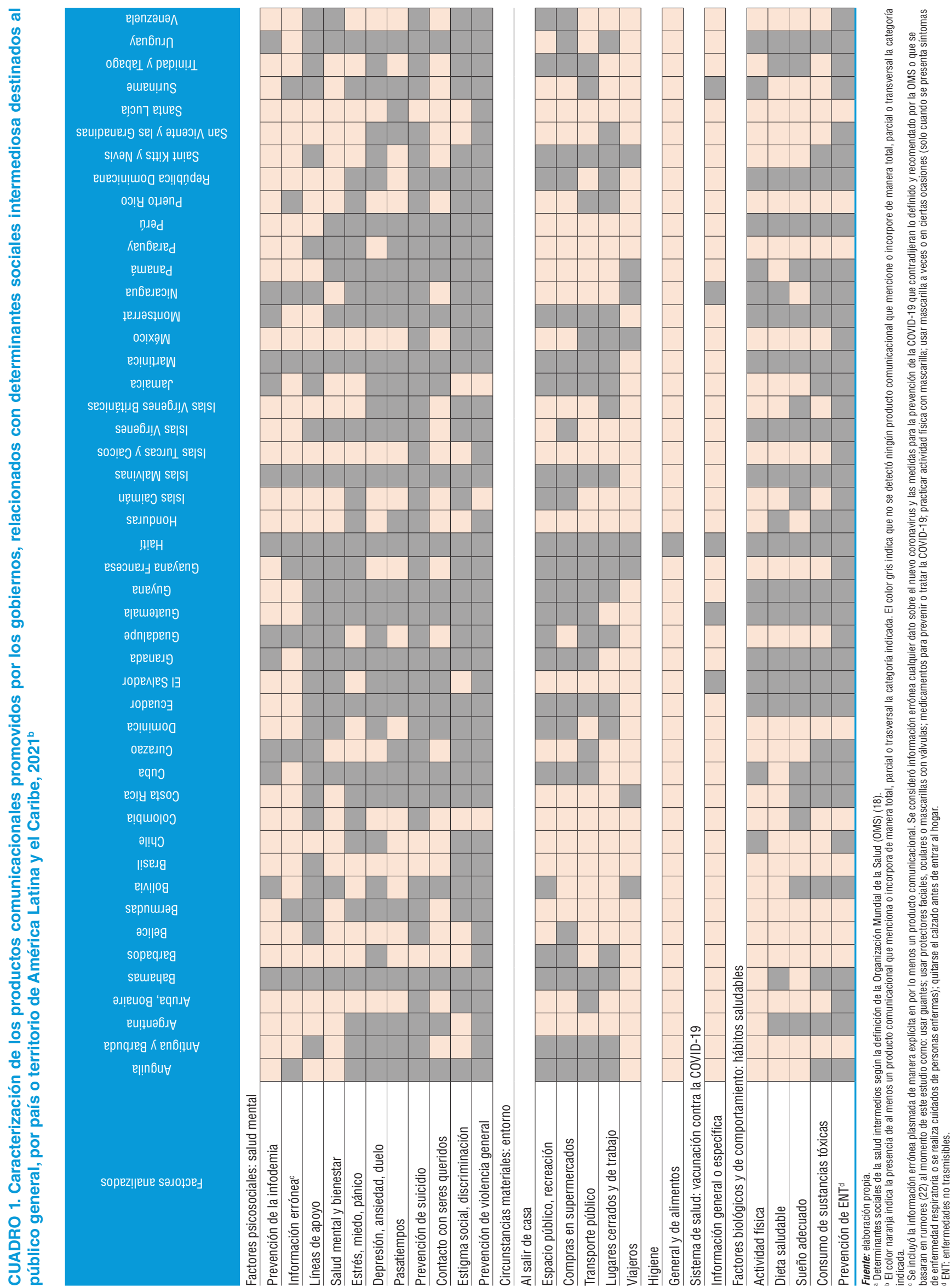


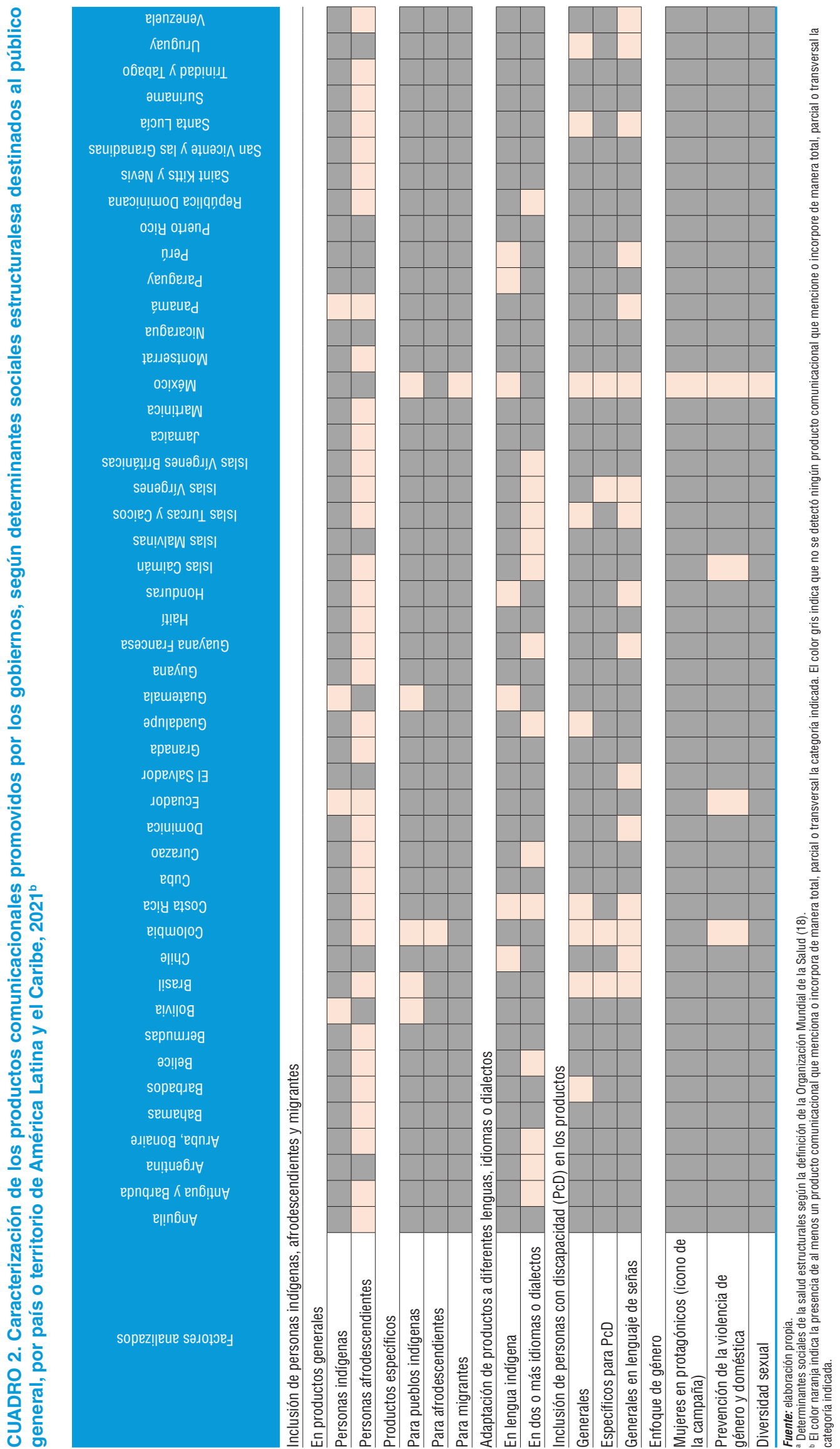


Se encontraron importantes vacíos en cuanto a las temáticas y el tratamiento transversal de la diversidad en los productos comunicacionales destinados a la población general, con escasa adaptación a diferentes lenguas - en un contexto en el que gran parte de los países de ALC cuentan con poblaciones indígenas (29) — ; esto confirma resultados similares encontrados en Taiwán (30).

Un hallazgo alarmante fue el escaso enfoque de género en los productos analizados, hecho ya descrito en parte en el estudio español (27). Estos factores - y otros abordados en este trabajo sobre el enfoque ajustado a la diversidad- desempeñan un papel clave en la aceptabilidad de la información y de las recomendaciones gubernamentales (31). Según otros autores (28) y las experiencias documentadas a partir de la epidemia de ébola (32), se requiere adaptar las intervenciones y los mensajes a los diferentes grupos y comunidades.

Como plantean Clark-Ginsberg y Petrun Sayers (12), la comunicación durante las crisis rara vez se adecua a satisfacer las necesidades de las poblaciones vulnerables. Sin embargo, estos grupos experimentan resultados aun más negativos, entre otras causas, por ser también más vulnerables a los efectos negativos de la infodemia que ha rodeado a la COVID-19.

Tanto en el presente estudio como en una investigación realizada en África (11), se encontraron mensajes con información no sustentada en datos científicos, en gran medida basados en rumores. Preocupa que una parte considerable de estos mensajes se publicaron en el 2020 y no se habían actualizado al revisarlos para esta investigación, una situación agudizada por los pocos productos informacionales específicos - o de mensajes dentro de productos generales- destinados a sensibilizar a la población sobre la importancia de combatir la infodemia. Este resultado concuerda con los de otro estudio realizado en países de América del Sur en el que se constata la falta de estrategias para enfrentar la infodemia desde los ministerios de salud de los países estudiados (33). Esta realidad debe ser objeto prioritario de futuros estudios y acciones.

A partir de los resultados y el análisis realizado, se proponen las siguientes recomendaciones de comunicación destinadas a los gobiernos de la Región para aplicar durante la pandemia de COVID-19.

\section{Políticas y estrategias de comunicación}

- Elaborar un plan de comunicación actualizado sobre la COVID-19, con un componente de monitoreo ajustado tanto a las diferentes etapas de la pandemia en cada país como al avance del conocimiento en el mundo.

- Desarrollar estrategias que incluyan productos comunicacionales diseñados desde un enfoque de los DSS (18) para contribuir a la mejor recepción, decodificación, aceptación y adopción de los mensajes y, a su vez, promover mejores prácticas de prevención y cuidados.

- Incluir en la política un componente de comunicación de riesgo dirigido a la prevención de la infodemia (8), de manera trasversal, en todas las etapas de la pandemia.

\section{Medios de difusión}

- Consolidar portales específicos para la COVID-19, cuyos mensajes, materiales y productos informacionales se mantengan actualizados y accesibles, y realizar un correcto ordenamiento de los mensajes según el público al que va dirigido, con una adecuada diferenciación de la información destinada al público general.

- Generar una estrategia unificada de publicación y amplificación entre los sitio web oficiales de salud y las redes sociales, a fin de lograr un mayor y mejor alcance de la información; este aspecto es importante, ya que resulta llamativo que en 10 de los países y territorios analizados no se halló información en los sitios web gubernamentales y se tuvo que recurrir a la red social Facebook para encontrarla.

\section{Contenido de los productos comunicacionales}

- Revisar y ajustar los productos existentes y sus contenidos - en lugar de crear nuevos-, algo que podría mejorarse en los 47 países y territorios estudiados.

- Analizar los productos disponibles para evaluar si están diseñados desde un enfoque de comunicación de riesgo y de los DSS (18) y adaptados a las particularidades de los diferentes grupos de la población.

- Difundir mensajes mediante productos orientados a las necesidades de las personas y evitar que contengan información falsa o errónea.

- Elaborar productos generales aptos para todos los grupos poblacionales; si bien en este estudio no se analizó el efecto positivo que podría generar la alfabetización en salud (9) para la correcta recepción de los mensajes por parte de los destinatarios, esto deberá tomarse en cuenta dada la cantidad de productos encontrados basados en videos largos (de más de 30 segundos) e infografías, en comparación con otros recursos más sencillos como spots e imágenes, que son más fáciles de comprender por una mayor parte de la población.

$\mathrm{Al}$ analizar estas recomendaciones, se deben tomar en cuenta algunas limitaciones de este estudio, en particular su alcance concreto y que no se tomó en cuenta el posible paralelismo diferencial entre el contenido de los productos comunicacionales y el contexto epidemiológico, las diferentes etapas de avance de la pandemia y las medidas tomadas por los gobiernos en cada país.

Ciertos productos y mensajes de comunicación creados y difundidos por los ministerios de salud pudieron no llegar a registrarse o no estar disponibles en línea al momento de este estudio. Además, en este trabajo no se analizaron los productos difundidos por otras vías de comunicación, como la prensa escrita, la radio, la televisión y varias redes sociales. Para evitar o reducir este posible sesgo, en los casos de mínima o ninguna presencia de los mensajes buscados en las páginas web gubernamentales, se recurrió a la red social de Facebook de las entidades oficiales de salud.

Por otra parte, no se indagó qué tipo de productos de comunicación fueron efectivamente asimilados por parte de las personas a las que iban dirigidos, por lo que se requieren estudios que profundicen en la recepción de la información, en consonancia con la alfabetización en salud (9).

A pesar de esas limitaciones se logró caracterizar de manera global el contenido de los productos y mensajes comunicacionales dirigidos a la prevención de la COVID-19 impulsados por los gobiernos de ALC, a partir de una búsqueda suficientemente 
exhaustiva y representativa de todos sus países y territorios, un tema con muy pocos antecedentes.

Se puede concluir que, si bien se encontró una cantidad considerable de productos comunicacionales para la prevención de la COVID-19, estos no reflejaban un adecuado enfoque de diversidad y salud integral, además de detectarse en los mismos vacíos y errores.

Se requiere con urgencia que las autoridades gubernamentales y sanitarias de ALC revisen sus políticas de comunicación sobre la COVID-19 a fin de lograr un mayor y mejor alcance de sus mensajes a todos los sectores de la población. Se deben recoger datos sistemática y longitudinalmente para capturar las variaciones en el comportamiento de la pandemia y ajustar los mensajes consecuentemente.

Estas recomendaciones pueden servir, debidamente ajustadas, para la formulación de políticas y estrategias de comunicación en futuras pandemias y crisis sanitarias en la Región.
Contribución de los autores. DM y LLM concibieron el estudio original y recolectaron los datos. DM analizó los datos, interpretó los resultados y redactó la primera versión del manuscrito. Ambas autoras revisaron todas las versiones del manuscrito y aprobaron la versión final.

Agradecimientos. A María Olivera Mores y Manuel Alemán por la revisión de estilo.

\section{Conflictos de intereses. Ninguno.}

Declaración. Las opiniones expresadas en este artículo son responsabilidad de las autoras y no necesariamente reflejan las opiniones, políticas o posiciones oficiales de las instituciones a las cuales están afiliadas, ni los criterios ni la política de la Revista Panamericana de Salud Pública / Pan American Journal of Public Health y/o de la Organización Panamericana de la Salud.

\section{REFERENCIAS}

1. Organización Panamericana de la Salud. Informes de situación de la COVID-19 [Internet]. Washington, DC: OPS; 2020 [acceso el 25 de mayo del 2021]. Disponible en: https://www.paho.org/es/ informes-situacion-covid-19

2. Abramo L, Cecchini S, Ullmann H. Enfrentar las desigualdades en salud en América Latina: el rol de la protección social. Cienc Saude Colet. 2020;25(5):1587-98.

3. Sánchez-Torres DA. Accesibilidad a los servicios de salud: debate teórico sobre determinantes e implicaciones en la política pública de salud. Rev Med Inst Mex Seguro Soc. 2017:55(1).

4. Benski C, Goto A, Creative Health Teams, Reich MR. Developing health communication materials during a pandemic. Front Commun. 2020;5:603656. https://doi.org/10.3389/fcomm.2020.603656

5. Finset A, Bosworth H, Butow P, Gulbrandsen P, Hulsman RL, Pieterse $\mathrm{AH}$, et al. Effective health communication-a key factor in fighting the COVID-19 pandemic. Patient Educ Couns. 2020;103:873-6. doi: 10.1016/j.pec.2020.03.027

6. Organización Panamericana de la Salud. Entender la infodemia y la desinformación en la lucha contra el COVID-19. Washington, DC: OPS; 2020 [acceso el 22 de mayo del 2021]. Disponible en: https:// iris.paho.org/bitstream/handle/10665.2/52053/Factsheet-Infodemic_spa.pdf?sequence $=14 \&$ isAllowed $=\mathrm{y}$

7. Abrams EM, Greenhawt M. Risk communication during COVID19. J Allergy Clin Immunol Pract. 2020;8(6):1791-4. doi: 10.1016/j. jaip.2020.04.012

8. World Health Organization. Emergencies: Risk communication [Internet]. Geneva: WHO; 2020 [acceso el 22 de mayo del 2021]. Disponible en: https://www.who.int/news-room/q-a-detail/ emergencies-risk-communication

9. Goto A. Communicating health information with the public: Lessons learned post disaster. J Glob Health Sci. 2020;2(1):e6. doi. org/10.35500/jghs.2020.2.e6

10. Mheidly N, Fares J. Leveraging media and health communication strategies to overcome the COVID-19 infodemic. J Public Health Policy. 2020;41(4):410-20. doi: 10.1057/s41271-020-00247-w

11. Seytre B. Erroneous communication messages on COVID-19 in Africa. Am J Trop Med Hyg. 2020;103(2):587-9.

12. Clark-Ginsberg A, Petrun Sayers EL. Communication missteps during COVID-19 hurt those already most at risk. J Conting Crisis Manage. 2020;1:1-6.

13. Karamouzian M, Johnson C, Kerr T. Public health messaging and harm reduction in the time of COVID-19. Lancet Psychiatry. 2020;7(5):390-1.

14. Rudd RE, Comings JP, Hyde JN. Leave no one behind: Improving health and risk communication through attention to literacy. J Health Commun. 2003;8(1):104-15.
15. Toppenberg-Pejcic D, Noyes J, Allen T, Alexander N, Vanderford M, Gamhewage G. Emergency risk communication: Lessons learned from a rapid review of recent gray literature on Ebola, Zika, and yellow fever. Health Commun. 2019;34(4):437-55. doi: 10.1080/10410236.2017.1405488

16. Venkatasubramanian M. Applications of qualitative content analysis: Evaluating the reliability and quality of health information websites. Qual Res Rep Commun. 2020:1-8.

17. Organización Mundial de la Salud. Brote de enfermedad por coronavirus (COVID-19): orientaciones para el público [Internet]. Ginebra: OMS; 2020 [acceso el 14 de mayo del 2021]. Disponible en: https:/ / www.who.int/es/emergencies/diseases/novel-coronavirus-2019/ advice-for-public

18. World Health Organization. A conceptual framework for action on the social determinants of health. Geneva: WHO; 2010 [acceso el 22 de mayo del 2021]. Disponible en: https://apps.who.int/iris/ handle/10665/44489

19. Organización de las Naciones Unidas para la Alimentación y la Agricultura. América Latina y el Caribe [Internet]. Roma: FAO; 2020 [acceso el 22 de mayo del 2021]. Disponible en: http://www.fao. org/3/v8300s/v8300s0o.htm

20. Carter N, Bryant-Lukosius D, DiCenso A, Blythe J, Neville AJ. The use of triangulation in qualitative research. Oncol Nurs Forum. 2014;41(5):545-7.

21. Organización Panamericana de la Salud. Gestión de la información y comunicación en emergencias y desastres: guía para equipos de respuesta. Washington, DC: OPS; 2009 [acceso el 22 de mayo del 2021]. Disponible en: https://www.paho.org/chi/dmdocuments/ GestionDeInformComunica_LowRes\%20Dic\%2009.pdf

22. Organización Mundial de la Salud. Consejos para la población acerca de los rumores sobre el nuevo coronavirus (2019-nCoV) [Internet]. Ginebra: OMS; 2020 [acceso el 5 de junio del 2021]. Disponible en: https:/ / www.who.int/es/emergencies/diseases/novelcoronavirus-2019/advice-for-public/myth-busters

23. Arocena F, Sobottka EA. Diversidad cultural en América Latina. Civitas (Porto Alegre, Brasil). 2017;17(2):205-9. doi: https://doi. org/10.15448/1984-7289.2017.2

24. Val Cubero A. La diversidad cultural: ¿es posible su aplicación al sector audiovisual? Comun Soc. 2017;(28):111-30.

25. MacNamara J. Media content analysis: Its uses, benefits and best practice. Methodol Asia Pacific Public Relat J. 2005;6(1):1-34.

26. Guzmán do Nascimento B. Comunicación y salud: la gestión de la crisis del ébola a través de las redes sociales. Rev Esp Comun Salud. 2018;9(2),196-202. https://doi.org/10.20318/recs.2018.4497

27. Villegas-Tripiana I, Villalba-Díaz A, López-Villegas A. Análisis de la información sobre COVID-19 en sitios web de organizaciones 
públicas sanitarias. Rev Esp Comun Salud. 2020;(Supl1):235-42. https:/ / doi.org/10.20318/recs.2020.5437

28. Mheidly N, Fares J. Leveraging media and health communication strategies to overcome the COVID-19 infodemic. J Public Health Policy. 2020;41(4):410-20. doi:10.1057/s41271-020-00247-w

29. Comisión Económica para América Latina y el Caribe. Los pueblos indígenas en América Latina [Internet]. México, DF: CEPAL; 2021 [acceso el 7 de junio del 2021]. Disponible en: https://www.cepal. $\mathrm{org} / \mathrm{fr} /$ infografias/los-pueblos-indigenas-en-america-latina

30. Chen CM. Public health messages about COVID-19 prevention in multilingual Taiwan. Multilingua. 2020;39(5):597-606.

31. Reddy BV, Gupta A. Importance of effective communication during COVID-19 infodemic. J Family Med Prim Care. 2020;9:3793-6.
32. Afolabi MO, Folayan MO, Munung NS, Yukubu A, Ndow G, Iegede A, et al. Lessons from the Ebola epidemics and their applications for COVID-19 pandemic response in sub-Saharan Africa. Dev World Bioeth. 2021;21(1):25-30. doi:10.1111/dewb.12275

33. Haraki CAC. Estratégias adotadas na América do Sul para a gestão da infodemia da COVID-19. Rev Panam Salud Publica. 2021;45:43. https://doi.org/10.26633/RPSP.2021.43

Manuscrito recibido el 30 de marzo del 2021. Aceptado para publicación, tras revisión, el 20 de julio del 2021.

\section{Communication products for COVID-19 prevention promoted by governments in Latin America and the Caribbean}

ABSTRACT Objective. Characterize the content of communication products for COVID-19 prevention promoted by governments in Latin America and the Caribbean (LAC) for the general public.

Methods. A descriptive, cross-cutting, and exploratory study of the communication products for COVID-19 prevention published up to 28 February 2021 on official websites of the ministries of health of the 47 countries and territories of LAC. Quantitative and qualitative methods based on the dimensions recommended by the World Health Organization (WHO) were employed, together with a social determinants of health (SDH) approach. The content analysis technique and investigator triangulation were used to improve internal validity. Results. 3253 information products were analyzed. In 47 countries and territories in LAC at least one product included a WHO recommendation; and in 46 at least one category or subcategory related to intermediate SDHs was addressed. Regarding structural SDHs, a gender approach was used in at least one product in only four countries, and an indigenous language in only seven countries. In 35 countries, at least one product was found to have erroneous information and in 13 countries the infodemic was not addressed.

Conclusions. A substantial number of communication products for COVID-19 prevention were found, but they lacked an adequate diversity approach and comprehensive health approach, and they showed gaps and communication errors. The countries should review their health communication policies in the context of the pandemic.

Keywords Health communication; coronavirus infections; government programs; Latin America; Caribbean region. 


\section{Produtos de comunicação para a prevenção da COVID-19 promovidos pelos governos da América Latina e do Caribe}

RESUMO

Objetivo. Caracterizar o conteúdo dos produtos de comunicação dirigidos à população em geral, para a prevenção da COVID-19, promovidos pelos governos da América Latina e do Caribe (ALC).

Métodos. Estudo descritivo, transversal e exploratório dos produtos de comunicação publicados até 28 de fevereiro de 2021 nos sites oficiais dos ministérios da Saúde de 47 países e territórios da ALC para a prevenção da COVID-19. Foram utilizados métodos quantitativos e qualitativos com base nas dimensões recomendadas pela Organização Mundial da Saúde (OMS) e enfoque nos determinantes sociais da saúde (DSS). Foram utilizadas as técnicas de análise de conteúdo e triangulação de investigador.

Resultados. Foram analisados 3253 produtos de informação. Nos 47 países e territórios da ALC, pelo menos um produto incluiu alguma recomendação da OMS, e em 46, foram abordadas algumas das categorias e subcategorias relacionadas aos DSS intermediários. Dos DSS estruturais, foram utilizados enfoque de gênero e alguma língua indígena em pelo menos um produto de apenas 4 e 7 países, respectivamente. Em 35 países foi encontrado pelo menos um produto com informações errôneas, e em 13 não foi abordada a infodemia.

Conclusões. Foi encontrado um número considerável de produtos de comunicação para a prevenção da COVID-19; entretanto, eles não refletiam uma abordagem adequada à diversidade e à saúde integral, e apresentavam lacunas e erros de comunicação. Os países devem revisar suas políticas de comunicação em saúde no contexto da pandemia.

Palavras-chave Comunicação em saúde; infecções por coronavirus; programas governamentais; América Latina; região do Caribe. 